\title{
Déploiement du e-learning en sciences de l'éducation : état des lieux en France en 2006
}

\section{Christophe Col}

UFR des sciences de l'éducation, Université Charles-de-Gaulle-Lille 3, FRANCE

christophe.col@hotmail.fr

\section{Fabien Fenouillet}

Université Paris X Nanterre, FRANCE

fabienfenouillet@yahoo.fr

\section{Recherche}

\section{Résumé}

Cet article présente un état des lieux du e-learning en sciences de l'éducation dans les universités françaises. L'exhaustivité étant le point de départ de notre méthodologie, une partie du travail a consisté à réunir des informations ciblées, d'autant plus difficiles à recueillir qu'elles apparaissaient aux yeux de certains responsables de dispositifs e-learning comme des renseignements sensibles. Nous avons comparé les différentes offres $e$-learning autour de trois grands axes d'interrogation : quelles universités proposent un dispositif dans le cadre des sciences de l'éducation? Quelles sont les ingénieries pédagogiques élaborées? Combien d'UE, d'étudiants et d'enseignants sont mobilisés? Les résultats obtenus en sciences de l'éducation n'indiquent pas l'émergence d'un modèle général d'enseignement, mais rappellent la part d'héritage de la formation à distance dans les dispositifs e-learning actuels.

\section{Abstract}

This article aims to provide a fairly exhaustive account of how e-learning has been (and continues to be) implemented in French universities, particularly in the field of educational sciences. We needed to collect targeted data to achieve our objective, although it should be mentioned that the e-learning program directors contacted were not necessarily ready to provide this information. To render the data as impartial as possible, we used a step-by-step verification process based on a triangular comparison of the information gathered through the interviews. We compared and contrasted different university e-learning programs. Three main questions guided our inquiry: What universities currently offer e-learning options in the educational sciences? What are the objectives, conditions and resources of these various programs, and how are they organized? How many credits, students and teachers do these programs represent? Our results do not indicate the emergence of any general teaching model in current e-learning systems used in the educational sciences, but they do bring to mind certain practices inherited from various models of distance learning. 


\section{Introduction}

Depuis quelques années, les nouvelles technologies de l'information et de la communication (TIC) prennent une place de plus en importante dans l'enseignement, notamment celui du supérieur. Cette utilisation des moyens modernes de communication à des fins d'enseignement s'inscrit dans une histoire riche et variée qui a traversé toutes les époques depuis la fin du xIxe siècle (Albéro, 2004; Russell, 2001). L'irruption d'Internet jusque dans le quotidien de l'homme moderne ne pouvait épargner le secteur de l'éducation en général ni celui de l'enseignement universitaire des sciences de l'éducation en particulier.

Même si de nombreux universitaires, étudiants ou médias semblent toujours et encore s'émerveiller des incroyables possibilités qu'offre Internet dans l'enseignement, cette utilisation commence à «dater » d'une dizaine d'années. Le terme d'e-learning, importation directe du monde anglosaxon, caractérise cette utilisation moderne des technologies pour diffuser les enseignements universitaires. Cependant, ce terme recouvrant des réalités et des utilisations d'une très grande variété, il semble indispensable de s'y arrêter quelques instants afin d'en circonscrire l'étendue sémantique dans le cadre de cet article.

L'adoption du terme e-learning a été fulgurante en France et semble liée à de nombreux facteurs, dont l'absence d'antécédents clairement identifiables (il ne s'agit pas par exemple uniquement d'enseignement assisté par ordinateur, d'hypertexte ou de multimédia), et son émergence tout aussi rapide dans un cadre commercial très friand de « technominalisme » anglo-saxon. Les publications scientifiques en langue anglaise sont très loin de cet hégémonisme puisqu'en fonction des auteurs il est possible, sans être exhaustif, de rencontrer une terminologie plus riche avec une orientation plus précise en fonction du terme employé. Voici quelques exemples d'utilisation avec, entre parenthèses, une tentative de traduction permettant d'apprécier le champ d'application pour la forme de e-learning ainsi décrite : computer-based learning (enseignement as- sisté par ordinateur), Internet learning (apprentissage via Internet), online learning (apprentissage en ligne), distributed learning (apprentissage distribué), networked learning (apprentissage en réseau), telelearning (téléapprentissage), virtual learning (apprentissage virtuel), computer-assisted learning (apprentissage assisté par ordinateur), Web-based learning (apprentissage web), Web-based training (formation web), distance learning (apprentissage à distance), asynchronous learning network (apprentissage asynchrone), learning object download (apprentissage par objet téléchargeable). Bien entendu, chaque terme nécessiterait d'être davantage explicité tant les nuances et les distinctions sont importantes dans le cadre de l'utilisation des technologies en enseignement. Cependant, un tel détail dépasserait le cadre de cet article et finirait par en diluer l'objet central. En 2000, la Commission européenne définit le e-learning comme « l'utilisation des nouvelles technologies multimédias et de l'Internet, pour améliorer la qualité de l'apprentissage en facilitant l'accès à des ressources et des services, ainsi que des échanges et la collaboration à distance ». Cette définition repose sur la foi technophile des décideurs politiques en matière d'enseignement, un enseignement qui devient de plus en plus central dans le développement économique des pays développés. Cependant, force est de constater que si quelques dispositifs e-learning semblent bien améliorer 1'apprentissage (Spencer et Hiltz, 2001), différentes analyses indiquent que le e-learning, sous diverses définitions, n'a pas pour effet d'augmenter ou de diminuer la qualité de l'apprentissage (Cavanaugh, Gillan, Kromrey, Hess et Blomeyer, 2004; Fenouillet et Déro, 2006). Dans certaines conditions, il apparaît même que l'utilisation des didacticiels et tutoriels peut avoir un impact positif indépendant de l'utilisation d'Internet (Cavanaugh, 2001; Olson et Wisher, 2002; Waxman, Lin et Michko, 2003). La définition européenne du e-learning met en avant la possible amélioration des apprentissages liés à son utilisation. Cependant, comme nous venons de le voir, il semble que cette amélioration, si elle existe, ne puisse être directement imputée au e-learning. Ce dernier rassemble en son sein différentes technologies et diverses potentialités qu'il convient dans un premier temps d'éclaircir. 
Le e-learning admet deux grandes catégories bipolaires : d'une part l'apprentissage en ligne ou hors ligne et d'autre part l'apprentissage autonome ou collaboratif. Le e-learning repose généralement sur la combinaison de ces deux niveaux. Par exemple, un enseignant peut commencer son cours en mettant en ligne un exerciseur nécessitant la collecte d'informations sur des sites web; ensuite, il peut décider de mettre à disposition des ressources dans un cours téléchargeable en ligne. Dans le même temps, cet enseignant peut proposer des réunions en ligne par clavardage (chat en anglais) pour éclaircir certains concepts. Enfin, les étudiants peuvent poser en retour des questions sur le cours, sur l'organisation de l'enseignement, ou encore sur les modalités de contrôle dans un forum de discussion.

Toutes les modalités du e-learning ont en commun l'utilisation incontournable d'Internet. Dans le même temps, elles incorporent des pans relativement anciens de technologie informatique, notamment sur l'enseignement assisté par ordinateur (EAO, EIAO ou CBI, CBL, CBT) ou sur l'enseignement à distance (EAD). Le e-learning s'en différencie néanmoins principalement au travers des nouvelles modalités de communication synchrone et asynchrone qui modifient radicalement les niveaux et les enjeux de l'échange pédagogique. La définition que propose Ally (2004) définit l'apprentissage en ligne - qui s'applique au e-learning - par l'utilisation d'Internet qui permet d'accéder à des ressources pédagogiques, à des enseignants, à d'autres apprenants et à des supports, et ceci, durant un processus d'apprentissage, dans l'objectif d'acquérir des connaissances, des compétences et de l'expérience (traduction libre). Cette présentation dresse un paysage technologique qui offre un potentiel riche et varié en matière de dispositif de formation. Comme nous allons le voir plus loin, cet avantage est aussi un inconvénient majeur lorsque l'on cherche à avoir une vision simple des différents dispositifs existants. Parallèlement à un certain nombre de tensions et de difficultés initiales ${ }^{1}$, on observe aujourd'hui une articulation grandissante entre les technologies de l'Internet d'une part et leur réception d'autre part. Et ce, dans les deux sens : les potentialités technologiques investissent l'enseignement en présentiel, alors que la variété pédagogique imprègne les offres d'enseignement Internet. L'objectif ici n'est pas de dresser un panorama du e-learning dans l'université française, mais d'établir un état des lieux des pratiques et des usages, uniquement dans le cadre des sciences de l'éducation en France, en nous interrogeant sur les contours des dispositifs e-learning, leurs promoteurs et les publics visés.

\section{Méthodologie}

Notre premier objectif de recherche a été de viser l'exhaustivité des données recueillies pour l'année universitaire 2005-2006. Nous avons alors examiné les stratégies pour étayer notre comparaison en n'omettant aucune université française, aucune UFR (unité de formation et de recherche) ni département de sciences de l'éducation. Pour valider notre comparaison - une comparaison déterminée à la fois par un territoire (national), par un cadre institutionnel (les universités) et par un champ disciplinaire, nous avons dû éviter le biais d'un recueil restreint. Précaution d'autant plus importante qu'un quatrième paramètre d'importance est venu confirmer l'obligation de globalité : la taille de notre corpus, toutes les universités ne présentant pas une offre e-learning dans le domaine des sciences de l'éducation. En prolongement du premier, notre deuxième objectif a été de vérifier chaque information obtenue concernant la présence (ou l'absence) d'un dispositif e-learning dans chaque université. Enquêter sur les dispositifs e-learning en France ne semble pas devoir être encore tout à fait transparent et il convient alors de recouper l'information pour vérifier sa pertinence. Les raisons sont multiples : soit que certains interlocuteurs préfèrent retenir les données qu'ils possèdent (stratégies de développement en cours d'élaboration), soit que les interlocuteurs qui nous répondent n'ont pas en leur possession toutes les informations que nous réclamons (les dispositifs sont parfois élaborés entre plusieurs responsables, plusieurs niveaux d'enseignement, voire plusieurs organisations administratives). Enfin, parce qu'il n'existe pas de tableau de bord national des dispositifs universitaires en matière $e$-learning comme il peut en exister par ailleurs en éducation ${ }^{2}$, nous avons dû l'établir avec la rigueur qui sied aux défrichements. 
À un premier niveau de prospection, nous nous sommes intéressés aux moteurs de recherche. Sur les sites du ministère de l'Éducation nationale et du ministère délégué à l'Enseignement supérieur et à la Recherche, nous avons entré plusieurs mots clefs : «e-learning », « téléenseignement », « formation continue et professionnelle »; nous avons vérifié le lien, d'après le résumé (lorsque celui-ci se rapprochait de notre sujet, ou lorsqu'il était vague, voire manquant), jusqu'à la perte de pertinence ${ }^{3}$.Le deuxième niveau de prospection s'est intéressé à la liste des universités du ministère de l'Éducation nationale croisée avec celle de l'AECSE (Association des enseignants et chercheurs en sciences de l'éducation). Le troisième niveau de prospection a retenu les requêtes de sites spécialisés : l'OFIVE (Observatoire des formations, de l'insertion et de la vie étudiante de Lille 3), les CTE-CTU (centres de téléenseignement universitaire), les SED et SEAD (services d'enseignement à distance), les services de formation continue et les services de visioconférence. Nous avons également retenu la FIED (Fédération interuniversitaire de l'enseignement à distance) et le CNED (Centre national d'enseignement à distance). Enfin, sur le site du ministère délégué à l’Enseignement supérieur («FormaSup, le guide de la formation à distance »), nous avons vérifié les liens obtenus de 1'AFPA (Association nationale de la formation professionnelle des adultes; pas de e-learning en sciences de l'éducation), du CNAM (Conservatoire national des arts et métiers; pas de e-learning en sciences de l'éducation) et du GRETA (Groupement d'établissements publics locaux d'enseignement; pas de sciences de l'éducation).
Notre objectif d'exhaustivité nous obligeant à recueillir l'ensemble des informations relatives à notre champ d'investigation, nous avons sollicité les responsables des dispositifs concernés en leur offrant plusieurs moyens de nous répondre : téléphone, courrier, renseignement de l'enquête en ligne.Le modèle du questionnaire a été retenu. Nous l'avons élaboré en définissant nos thèmes de recherche, puis en les déclinant en questions, elles-mêmes subdivisées en items. Chaque version du questionnaire a été testée. Nous avons arrêté notre construction sur la quatrième version. Celle-ci comporte 27 questions, parmi lesquelles 13 sont des questions fermées (réponse unique et/ou multiple) et 14 sont des questions ouvertes.Une fois que nous avons obtenu la liste complète des universités françaises proposant des dispositifs de formation e-learning en sciences de l'éducation, nous avons dû penser aux critères permettant non pas de les départager, mais d'appréhender finement leurs modalités d'organisation en vue de les comparer. Les critères que nous avons retenus sont de trois ordres : 1) des critères quantitatifs (nombre d'étudiants concernés par le dispositif e-learning, nombre d'unités d'enseignement (UE), ou encore nombre de niveaux préparés); 2) des critères pédagogiques (présence d'un tutorat par exemple, ou critères de sélection des étudiants pour qu'ils intègrent le dispositif); 3) des critères morphologiques (formes d'enseignement prises par chaque dispositif e-learning notamment: du "présentiel enrichi » ${ }^{4}$ à un «présentiel quasi inexistant ${ }^{5}$ ).

Tableau 1. Les différentes familles de critères

\begin{tabular}{|c|c|c|c|}
\hline & Critères quantitatifs & Critères pédagogiques & Critères morphologiques \\
\hline Définition & $\begin{array}{l}\text { Détaillant les dimensions chiffrables dans } \\
\text { chaque dispositif }\end{array}$ & $\begin{array}{l}\text { Identifiant les objectifs pédagogiques } \\
\text { poursuivis par les responsables des } \\
\text { dispositifs }\end{array}$ & $\begin{array}{l}\text { Définissant les différentes formes des } \\
\text { dispositifs mis en place }\end{array}$ \\
\hline $\begin{array}{l}\text { Détail } \\
\text { des critères }\end{array}$ & $\begin{array}{l}\text { - Nombre d'étudiants; } \\
\text { - Nombre de niveaux; } \\
\text { - Nombre d'unités d'enseignement (UE); } \\
\text { - Nombre de diplômes préparés. }\end{array}$ & $\begin{array}{l}\text { - Présence d'une FAQ6; } \\
\text { - Moteur de recherche; } \\
\text { - Forum (nombre et organisation); } \\
\text { - Tutorat (nombre et organisation); } \\
\text { - Critères de sélection présidant à } \\
\text { l'inscription; } \\
\text { - Conception du dispositif (organisé } \\
\text { autour des cours; autour du forum; } \\
\text { autour des questions, etc.). }\end{array}$ & $\begin{array}{l}\text { - Année de création; } \\
\text { - Tests du dispositif; } \\
\text { - Reconduction du dispositif l'année } \\
\text { suivante; } \\
\text { - Suivi des cours (offre « mixte » ou } \\
\text { « tout à distance »); } \\
\text { - Type de dispositif (présentiel enrichi; } \\
\text { présentiel amélioré; présentiel allégé; } \\
\text { présentiel réduit; présentiel quasi } \\
\text { inexistant). }\end{array}$ \\
\hline
\end{tabular}


Tableau 2. Le e-learning en sciences de l'éducation (nombre d'universités)

\begin{tabular}{|c|c|c|}
\hline $\begin{array}{c}\text { Nombre total d'universités proposant des } \\
\text { sciences de l'éducation }\end{array}$ & $\begin{array}{c}\text { Nombre d'universités proposant des sciences } \\
\text { de l'éducation et un dispositif e-learning }\end{array}$ & $\begin{array}{c}\text { Pourcentage des universités proposant des } \\
\text { sciences de l'éducation et un dispositif } \\
\text { e-learning sur le nombre total }\end{array}$ \\
\hline 32 & 8 & $25 \%$ \\
\hline
\end{tabular}

\begin{tabular}{|c|c|c|c|c|c|c|c|c|c|}
\hline \multirow{3}{*}{$\begin{array}{l}\text { Nom des universités proposant } \\
\text { des sciences de l'éducation et un } \\
\text { dispositif e-learning }\end{array}$} & \multirow{2}{*}{\multicolumn{3}{|c|}{$\begin{array}{l}\text { Étudiants inscrits } \\
\text { en sciences de } \\
\text { l'éducation } \\
\text { (année 2005-2006) }\end{array}$}} & \multirow{2}{*}{\multicolumn{3}{|c|}{$\begin{array}{c}\text { Étudiants inscrits en } \\
\text { e-learning } \\
\text { (année 2005-2006) }\end{array}$}} & \multicolumn{3}{|c|}{ Inscrits } \\
\hline & & & & & & & \multirow{2}{*}{$\begin{array}{c}\text { Total des } \\
\text { inscriptions }\end{array}$} & \multirow{2}{*}{$\begin{array}{c}\text { Inscriptions } \\
\text { e-learning }\end{array}$} & \multirow{2}{*}{$\begin{array}{l}\text { Pourcentage } \\
\text { des inscriptions } \\
e \text {-learning / tota } \\
\text { des inscriptions }\end{array}$} \\
\hline & L3 & M1 & M2 & L3 & M1 & M2 & & & \\
\hline $\begin{array}{l}\text { Besançon } 8 \\
\text { Université de Franche-Comté }\end{array}$ & 169 & 59 & - & $?$ & $?$ & $?$ & 228 & $?$ & $?$ \\
\hline Lille 1 USTL & 250 & 80 & 60 & - & - & 30 & 390 & 30 & $7 \%$ \\
\hline $\begin{array}{l}\text { Lille } 3 \\
\text { Charles-de-Gaulle }\end{array}$ & 1407 & 418 & 93 & 475 & 175 & - & 1918 & 650 & $33,8 \%$ \\
\hline Strasbourg Pasteur & 227 & 110 & 55 & - & - & 55 & 392 & 55 & $14 \%$ \\
\hline $\begin{array}{l}\text { Lyon } 2 \\
\text { Lumière }\end{array}$ & 1349 & 250 & 120 & 460 & 60 & - & 1719 & 520 & $30,2 \%$ \\
\hline $\begin{array}{l}\text { Montpellier } 3 \\
\text { Paul-Valéry }\end{array}$ & 475 & 97 & 70 & 150 & - & - & 642 & 150 & $23,5 \%$ \\
\hline $\begin{array}{l}\text { Paris } 8^{9} \\
\text { Vincennes-Saint-Denis }\end{array}$ & $?$ & $?$ & ? & $?$ & $?$ & $?$ & ? & $?$ & $?$ \\
\hline Paris X Nanterre & 419 & 229 & 120 & 200 & 100 & 50 & 768 & 350 & $45,5 \%$ \\
\hline $\begin{array}{l}\text { Rouen } \\
\text { Université de Rouen }\end{array}$ & 1031 & 142 & 190 & 500 & 34 & 140 & 1363 & 674 & $49,5 \%$ \\
\hline
\end{tabular}

\begin{tabular}{|c|c|c|c|c|c|}
\hline & \multirow{2}{*}{$\begin{array}{l}\text { Nombre d'UE pour l'année en } \\
\text { cours (2005-2006) }\end{array}$} & \multirow{2}{*}{$\begin{array}{l}\text { Nombre d'UE pour l'année } \\
\text { prochaine (2006-2007) }\end{array}$} & \multicolumn{3}{|c|}{ Diplôme(s) préparé(s) } \\
\hline & & & L3 & M1 & M2 \\
\hline Besançon & $\begin{array}{l}26 \text { ( } 10 \text { en L3; } 10 \text { en M1; } \\
6 \text { en M2) }\end{array}$ & $?$ & oui & oui & oui \\
\hline Lille 1 & 13 (12 en M2 + 1 stage) & 14 (13 en M2 + 1 stage) & non & non & oui \\
\hline Lille 3 & $\begin{array}{l}60 \text { (entre } 20 \text { et } 30 \text { en L3; entre } 20 \\
\text { et } 30 \text { en M1) }\end{array}$ & $\begin{array}{l}60 \text { (entre } 20 \text { et } 30 \text { en L3; entre } 20 \\
\text { et } 30 \text { en M1) }\end{array}$ & oui & oui & non \\
\hline Strasbourg Pasteur & 13 (en M21) & 15 (en M2) & non & non & oui \\
\hline Lyon 2 & $\begin{array}{l}5 \text { (en L3) } \\
\text { et } 2 \text { (en M1) }\end{array}$ & 5 (en L3) et 4 (en M1) & oui & oui & non \\
\hline Montpellier 3 & 12 (en L3) & 12 (en L3) & oui & non & non \\
\hline Paris 8 & 5 (en L3) & & oui & non & non \\
\hline Paris X Nanterre & 6 (5 en L3; 1 en M1) & 7 (6 en L3; 1 en M1) & oui & oui & oui \\
\hline Rouen & $\begin{array}{l}52 \text { (20 environ en L3; } \\
12 \text { environ en M1; } \\
20 \text { environ en M2) }\end{array}$ & $\begin{array}{l}52 \text { (20 environ en L3; } \\
12 \text { environ en M1; } \\
20 \text { environ en M2) }\end{array}$ & oui & oui & oui \\
\hline
\end{tabular}




\section{Résultats}

Il convient de relever, en premier lieu, que les dispositifs e-learning sont conçus comme des dispositifs de formation à part entière par leurs initiateurs, et qu'ils ne sont en aucun cas considérés comme des propositions optionnelles d'enseignement. Les données chiffrées qui sont présentées vont permettre de découvrir et de distinguer l'ampleur et la diversité du dispositif e-learning. Nous relevons déjà qu'un quart des universités enseignant les sciences de l'éducation proposent un dispositif $e$-learning à leurs étudiants (voir le Tableau 2). Nous notons ensuite que, pour chaque université, la part des étudiants concernés par ces dispositifs représente environ un tiers du total des étudiants inscrits en sciences de l'éducation.Les dispositifs e-learning sont répartis dans 8 universités sur les 32 qui proposent des sciences de l'éducation en France (voir le Tableau 2). Pour cette année 2005-2006, un quart des universités proposent donc un dispositif technique permettant de faire du e-learning en sciences de l'éducation. Ce pourcentage est en constante évolution. En effet, la plupart des dispositifs tels qu'ils sont conçus et proposés aujourd'hui n'existaient pas encore il y a trois ans. Ceux-ci ne

\begin{tabular}{|c|c|c|c|c|}
\hline & $\begin{array}{l}\text { Mise en place } \\
\text { d'un tutorat }\end{array}$ & Type de tutorat & $\begin{array}{l}\text { Présence d'un } \\
\text { forum }\end{array}$ & Type de forum \\
\hline Lille 1 & oui & $\begin{array}{l}\text { - Un tutorat par UE géré par } \\
\text { chaque enseignant; } \\
\text { - Un tutorat collectif géré par des } \\
\text { animateurs de plate-forme. }\end{array}$ & oui & $\begin{array}{l}\text { - Présence d'un forum par UE; } \\
\text { - Présence d'un forum commun à } \\
\text { l'ensemble des UE; } \\
\text { - Présence d'un forum réservé aux } \\
\text { étudiants (hors UE). }\end{array}$ \\
\hline Lille 3 & oui & $\begin{array}{l}\text { - Un tutorat par UE géré par } \\
\text { chaque enseignant. }\end{array}$ & oui & $\begin{array}{l}\text { - Présence d'un forum par UE; } \\
\text { - Présence d'un forum commun à } \\
\text { l'ensemble des UE; } \\
\text { - Présence d'un forum réservé aux } \\
\text { étudiants (hors UE); } \\
\text { - Présence d'un forum réservé aux } \\
\text { enseignants (hors UE). }\end{array}$ \\
\hline Strasbourg Pasteur & oui & $\begin{array}{l}\text { - Un tutorat par UE géré par les } \\
\text { tuteurs; } \\
\text { - Un tutorat par UE supervisé par } \\
\text { chaque enseignant. }\end{array}$ & oui & $\begin{array}{l}\text { - Présence d'un forum par UE; } \\
\text { - Présence d'un forum commun à } \\
\text { l'ensemble des UE; } \\
\text { - Présence d'un forum réservé aux } \\
\text { étudiants (hors UE); } \\
\text { - Présence d'un forum réservé aux } \\
\text { enseignants (hors UE). }\end{array}$ \\
\hline Lyon 2 & oui & $\begin{array}{l}\text { - Un tutorat collectif géré par les } \\
\text { administrateurs; } \\
\text { - Un tutorat collectif géré par des } \\
\text { animateurs de plate-forme; } \\
\text { - Un tutorat géré par le directeur } \\
\text { de mémoire (en Master } 1 \text { et/ou } \\
\text { Master 2). }\end{array}$ & oui & $\begin{array}{l}\text { - Présence d'un forum par UE; } \\
\text { - Présence d'un forum commun à } \\
\text { l'ensemble des UE. }\end{array}$ \\
\hline Montpellier 3 & oui & $\begin{array}{l}\text { - Un tutorat par UE géré par } \\
\text { chaque enseignant. }\end{array}$ & non & - $\square$ \\
\hline Paris 8 & oui & $\begin{array}{l}\text { Un tutorat par UE géré par chaque } \\
\text { enseignant; } \\
\text { Un tutorat collectif géré par des } \\
\text { animateurs de plate-forme. }\end{array}$ & oui & $\begin{array}{l}\text { Présence d'un forum par UE; } \\
\text { Présence d'un forum commun à } \\
\text { l'ensemble des UE; } \\
\text { Présence d'un forum «pause café » } \\
\text { (échange détente). }\end{array}$ \\
\hline Paris $\mathrm{X}$ & oui & $\begin{array}{l}\text { Un tutorat par UE géré par chaque } \\
\text { enseignant. }\end{array}$ & oui & Présence d'un forum par UE. \\
\hline Rouen & oui & $\begin{array}{l}\text { Un tutorat collectif géré par les } \\
\text { administrateurs; } \\
\text { Un tutorat collectif géré par des } \\
\text { animateurs de plate-forme; } \\
\text { Un tutorat géré par le directeur de } \\
\text { mémoire (en Master 1 et/ou Master } \\
\text { 2). }\end{array}$ & oui & $\begin{array}{l}\text { Présence d'un forum par UE, par } \\
\text { niveau d'études et par tutorat. }\end{array}$ \\
\hline
\end{tabular}


cessent de se développer, de se diversifier et d'être rejoints par de nouveaux dispositifs dans d'autres universités.

Dans certaines universités, une formation complète peut être proposée aux étudiants. C'est le cas notamment de 1'Université de Lille 3 qui offre une licence 3e année complète en e-learning : un étudiant peut actuellement suivre l'intégralité de sa licence par Internet (voir le Tableau 3).

Si nous croisons les résultats du Tableau 3 et du Tableau 4, nous nous rendons compte que le nombre d'UE concernées par chaque dispositif e-learning n'est pas corrélé avec le nombre d'étudiants inscrits, pas plus qu'il ne l'est avec les niveaux d'études préparées. En effet, ce n'est pas parce qu'un dispositif couvrira l'ensemble des niveaux de formation (Licence 3, Master 1 et Master 2) qu'il disposera inévitablement d'une palette large d'UE. C'est le cas, par exemple, des dispositifs de Paris X (6 UE) et de Rouen (50 UE), alors qu'ils couvrent l'un et l'autre les niveaux Licence 3, Master 1 et Master 2. D'autre part, un dispositif peut n'ouvrir que quelques UE tout en faisant le choix de les proposer à un grand nombre d'étudiants, alors qu'un autre dispositif fera le choix opposé. Dans le premier cas, on trouve Lyon 2, qui a ouvert 7 UE pour 520 étudiants. Dans le second cas, Montpellier 3 a fait le choix d'ouvrir 12 UE pour 150 étudiants et Strasbourg Pasteur a ouvert 13 UE pour 55 étudiants. Enfin, le nombre moyen d'étudiants est de 33,5 par UE (Lille 1, Lille 3, Montpellier 3 et Rouen sont en dessous de cette moyenne, tandis que Paris X et Lyon sont au-dessus).

Comme nous l'avons esquissé en introduction, le e-learning introduit des moyens de communication synchrone et asynchrone qui ont pour vocation de faciliter les échanges entre enseignants et étudiants. Cette possibilité est une innovation majeure qui le distingue d'autres dispositifs de formation relevant traditionnellement de l'enseignement à distance. Cependant, la disponibilité des outils synchrone et asynchrone ne rend pas compte nécessairement de leurs usages. Autrement dit, cet usage de la parole donnée aux étudiants peut dans certains dispositifs être totalement facultatif, alors qu'il est pour d'autres un vecteur clef de l'enseignement. Il n'existe malheureusement pas de nomenclature claire et univoque à ce niveau. C'est pourquoi il nous a semblé opportun d'essayer de rendre compte des outils et des formes d'utilisation pour donner au lecteur une représentation de l'utilisation du e-learning en sciences de l'éducation.

Le Tableau 5 nous permet de constater que si l'outil asynchrone que représente le forum est placé au centre de l'enseignement, sa gestion peut totalement différer d'une université à l'autre. Cette gestion différentielle d'un outil de ce type fait bien entendu écho aux pratiques pédagogiques, matière évanescente par excellence qu'il est tout aussi difficile d'appréhender que l'usage des outils de communication.

Les dispositifs e-learning sont élaborés selon des orientations pédagogiques multiples. Si toutes les plates-formes sont conçues pour que les documents (cours et textes) soient consultables et téléchargeables en ligne, d'autres procédés ont également été développés afin de faciliter l'exploration des niveaux d'échanges : enseignements élaborés en prolongement des cours, mais aussi enseignements construits autour des forums et des questionnements étudiants. Les plates-formes que nous avons pu voir utilisent des zones de fonctionnalités (zone permettant le téléchargement des cours, zone regroupant les fonctions communication synchrone et asynchrone...). C'est pour cette raison que nous utilisons le terme de zone dans le Tableau 6. C'est sans doute à ce niveau que les architectures techniques ont le plus de poids au regard de l'orientation pédagogique visée. Cependant, il est difficile de pousser ici plus loin l'analyse sans dépasser l'objet de ce présent article. En effet, certaines plates-formes peuvent également permettre des usages qui ne sont pas employés, car ils ne font pas partie des pratiques instaurées pour des raisons diverses et variées (voir Blandin, 2006, pour une discussion approfondie sur les implications techniques de l'usage des TIC).

Haeuw, Duveau-Patureau, Bocquet, Schaff et Roy-Picardi (2001), dans un document destiné à impulser la pénétration du e-learning dans l'enseignement supérieur français, indiquent 
Tableau 6. Les pratiques pédagogiques

\begin{tabular}{|c|c|c|c|c|c|}
\hline & $\begin{array}{l}\text { Enseignements } \\
\text { établis autour des } \\
\text { documents mis } \\
\text { en ligne (cours, } \\
\text { texte...) }\end{array}$ & $\begin{array}{l}\text { Enseignements } \\
\text { élaborés en } \\
\text { réponse aux } \\
\text { interrogations des } \\
\text { étudiants }\end{array}$ & $\begin{array}{l}\text { Enseignements } \\
\text { organisés autour } \\
\text { de la zone de cours }\end{array}$ & $\begin{array}{c}\text { Enseignements } \\
\text { organisés autour } \\
\text { du forum }\end{array}$ & $\begin{array}{l}\text { Autre élaboration des } \\
\text { enseignements }\end{array}$ \\
\hline Lille 1 & oui & non & non & oui & \\
\hline Lille 3 & oui & oui & oui & oui & \\
\hline Strasbourg Pasteur & oui & non & oui & oui & $\begin{array}{l}\text { - Construction d'activités } \\
\text { collectives autour de situations } \\
\text { problèmes; } \\
\text { - Les enseignants élaborent les } \\
\text { cours et conçoivent l'activité; } \\
\text { - Les tuteurs sont chargés } \\
\text { d'animer les activités. }\end{array}$ \\
\hline Lyon 2 & oui & non & non & non & \\
\hline Montpellier 3 & oui & non & non & non & \\
\hline Paris 8 & oui & non & non & oui & \\
\hline Paris $\mathrm{X}$ & oui & non & oui & non & \\
\hline Rouen & oui & non & oui & oui & $\begin{array}{l}\text { - Construction du cursus en ligne } \\
\text { par production des étudiants; } \\
\text { - En Licence } 3 \text { : modèle sommatif } \\
\text { et transmitif classique (donc } \\
\text { organisé autour des cours); } \\
\text { - En M1 ou M2 : cours utilisés } \\
\text { autrement, modèles variables, } \\
\text { plus de travail collaboratif. }\end{array}$ \\
\hline
\end{tabular}

différentes possibilités de mixage entre le présentiel et le tout à distance. Cette nomenclature permet d'avoir une autre idée de la diversité des usages pédagogiques liés au e-learning ainsi qu'une mesure indirecte de leur pénétration dans les enseignements, offrant un autre éclairage sur l'usage effectif du e-learning. Nous voyons ici apparaître deux dispositifs particuliers : Montpellier 3 et Paris X. La place prise par le $e$ learning à Montpellier 3 laisse penser que la configuration des enseignements change peu l'affectation des heures allouées au présentiel. Le dispositif de Paris X ne va pas jusqu'au bout des

\begin{tabular}{|c|c|c|c|c|c|}
\hline & \multicolumn{5}{|c|}{ Dispositif plus ou moins à distance ${ }^{10}$} \\
\hline & présentiel enrichi & $\begin{array}{l}\text { présentiel } \\
\text { amélioré }\end{array}$ & présentiel allégé & présentiel réduit & $\begin{array}{l}\text { présentiel quasi } \\
\text { inexistant }\end{array}$ \\
\hline Lille 1 & non & non & non & non & oui \\
\hline Lille 3 & non & non & oui & oui & oui \\
\hline Strasbourg Pasteur & non & non & non & non & oui \\
\hline Lyon 2 & non & non & oui & oui & non \\
\hline Montpellier 3 & oui & non & non & non & non \\
\hline Paris 8 & non & non & oui & oui & oui \\
\hline Paris $\mathrm{X}$ & non & non & oui & oui & non \\
\hline Rouen & non & non & oui & oui & oui \\
\hline
\end{tabular}




\begin{tabular}{|l|c|c|}
\hline \multirow{2}{*}{} & \multicolumn{2}{c|}{ Offre de formation } \\
\cline { 2 - 4 } & Mixte & An distance \\
\hline Lille 1 & oui & oui \\
\hline Lille 3 & non & oui \\
\hline Strasbourg Pasteur & oui & oui \\
\hline Lyon 2 oui & oui \\
\hline Montpellier 3 & oui & oui \\
\hline Paris 8 & oui & non \\
\hline Paris X & oui & oui \\
\hline Rouen & & \\
\hline
\end{tabular}

possibilités de mise à distance. Dans les deux cas, la référence au présentiel semble ne pas devoir être totalement effacée. Des enquêtes complémentaires seraient à mener pour analyser les représentations des enseignants et des étudiants vis-à-vis de l'impact qu'incarne encore l'enseignement présentiel. Dans ces deux cas, cette situation perdure au travers de contraintes spécifiques qu'il serait presque possible de qualifier d'originales. En ce qui concerne Paris X, la moitié des cours sont dispensés en présentiel alors que l'autre moitié est remplacée par un «équivalent » en e-learning. Dans ces conditions, si l'étudiant ne peut se rendre à l'université, il lui est impossible de suivre la totalité des enseignements. Montpellier pour sa part a choisi de « dédoubler » ses enseignements en e-learning, ce qui s'apparente donc à une forme de « remédiation » et ne dispense nullement l'étudiant de venir à l'intégralité des cours en présentiel.

Le dernier point concerne la possibilité de mixage qu'offre le e-learning entre des unités qui peuvent être suivies à distance et d'autres en présentiel. Cette possibilité de mixer les unités de formation permet aux étudiants de choisir les combinaisons qui leur conviennent le mieux. Comme nous le montre le Tableau 8, les solutions proposées aux étudiants par chaque organisation universitaire (département et/ou UFR de sciences de l'éducation) en matière de suivi des cours sont de forme "mixée ». Les étudiants peuvent choisir au cours de leur(s) cycle(s) de formation de suivre certaines UE en présentiel, et d'autres à distance via Internet. Ils composent ainsi leur propre participation aux cours, qui alterne entre présence et distance physique, au gré de leurs envies et de leurs obligations. Seul le dispositif de Lille 1 ne peut être suivi qu'à distance, tandis que celui de Paris $\mathrm{X}$ relaye au cours de l'année des UE à suivre en présentiel et à distance ${ }^{11}$.

La logique de développement du e-learning, sauf cas particulier, ne vise donc pas à remplacer le présentiel, mais à lui donner davantage de souplesse.

\section{Discussion}

Un rapport (U.S. Department of Education, National Center for Education Statistics, 2002) montre qu'un peu plus de $60 \%$ des étudiants à distance en 1999-2000 utilisaient Internet pour suivre leurs cours et ce chiffre atteignait près de $80 \%$ pour les étudiants en Master. Il est difficile en France de disposer de chiffres sur l'utilisation d'Internet, que les étudiants soient éloignés ou non de leurs lieux d'enseignement. Toutes les universités françaises possèdent un site Internet ou sont en passe de déployer des espaces numériques de travail. Parallèlement, tous les étudiants ont potentiellement accès aux nouvelles technologies pour suivre leurs cours. Mais qu'en est-il concrètement? Au travers d'un exemple (significatif tout au moins des universités de lettres), l'étude du déploiement du e-learning en sciences de l'éducation permet d'apporter quelques réponses à cette question. Il est à signaler qu'en France les sciences de l'éducation ont la particularité de rassembler toutes les disciplines qui ont pour ambition de mener des 
recherches en éducation (psychologie, sociologie, didactique, pédagogie...), mais qu'il s'agit en même temps d'une discipline à part entière comme peuvent l'être les sciences qui la composent. Les résultats de cette recherche font apparaître qu'un quart des universités qui proposent des sciences de l'éducation utilisent le e-learning pour dispenser leurs cours. En général, par rapport à l'offre présentielle, peu d'unités sont proposées en e-learning. Les quelques indications que nous avons tenté d'établir sur le plan pédagogique font apparaître que les universités utilisant le e-learning ont des pratiques pédagogiques très diverses, notamment sur les modalités d'utilisation des outils de communication.

Il est très difficile de connaître les pratiques pédagogiques qui se développent derrière les usages technologiques. C'est pour cette raison que des entretiens ont été menés auprès d'enseignants de l'Université Lille 3, afin de décrypter plus avant les pratiques effectives. Ils se déclarent ${ }^{12}$ plus libres d'inventer des pédagogies non frontales, que ce soit en Master 1 ou en Licence 3. La pédagogie leur apparaît de la sorte plus active en e-learning : les étudiants prennent connaissance du cours en début d'année, ce qui permet de déplacer significativement la part et la place du pédagogique. Échappant au tutorat qui encadre les cours en présentiel ${ }^{13}$, les enseignants considèrent que leur place se trouve plus décentrée vis-à-vis des savoirs. Ils se sentent plus à même de se concentrer sur les étudiants et les échanges. Dans ce contexte, une des variables les plus sensibles dans les discours est le temps. Alors qu'ils ne s'abritent nullement derrière la distanciation électronique pour se sentir affranchis de certaines contingences d'activité, les enseignants interrogés pensent au contraire que les enseignements qu'ils dispensent par Internet leur demandent plus de temps et de disponibilité qu'en présentiel. Si une majorité d'entre eux se rendent sur la plate-forme deux à trois fois par semaine, ils sont nombreux à le faire plus de quatre fois, voire tous les jours pour certains. Et chaque connexion prend, en moyenne, de 30 minutes à 2 heures. Quantitativement, la mise en place de cours par Internet demande un investissement plus important, l'activité en étant encore dans sa phase d'innovation. À terme, il est tout à fait pensable que la somme de travail allouée au e-learning s'équilibre avec celle que l'on relève actuellement en présentiel. Mais, pour l'instant, les enseignants notent une activité sur Internet plus conséquente, qui exige de leur part découverte et invention de formes nouvelles de production et d'échanges du savoir.

Inventer prend du temps, découvrir toutes les possibilités de la plate-forme également. Les exemples qui reviennent le plus fréquemment sont les exerciseurs ${ }^{14}$ et les questionnaires à choix multiple (QCM). Les exercices en ligne et les QCM sont pensés pour certifier un niveau et non pour évaluer les étudiants. Ils permettent de suivre les progressions et la maîtrise des connaissances. Le fait de moduler la durée pour renseigner en ligne les QCM ${ }^{15}$ permet par ailleurs de modifier les conditions d'exercice et d'obliger ainsi les étudiants à mobiliser plus ou moins rapidement leurs connaissances. Les connexions entre les champs disciplinaires et le fait de les présenter de telle manière qu'ils s'intègrent aux formes électroniques de partage des connaissances sont également cités. Certains enseignants parlent aussi de la complexité pédagogique représentée par la mise en ligne de textes, afin que cette mutualisation des savoirs reste profitable sans risque de tomber dans le pensum littéraire. Dans la zone d'échanges de documents électroniques, ils créent ainsi des « casiers » portant le nom de l'étudiant responsable, c'est-à-dire des dossiers dans lesquels les uns et les autres peuvent entrer et échanger des documents.Les échanges de documents des enseignants vers les étudiants se complètent d'échanges des étudiants vers les enseignants, ou entre étudiants. Cette " circulation circulaire » des informations est encouragée afin de mobiliser la participation des étudiants. L'ensemble des enseignants que nous avons interrogés ont insisté sur le fait que ce partage d'articles et de textes complémentaires favorisait l'implication des apprenants.Une autre pratique qui donne de très bons résultats consiste à demander à ceux qui le souhaitent qu'ils fassent des synthèses, des glossaires en vue d'être lus et utilisés par l'ensemble des étudiants travaillant dans l’UE. Cette délégation fonctionne très bien en ce qui concerne l'appropriation du cours. Autrement dit, l'élaboration et le partage des corpus sur lesquels chacun peut réfléchir sont des vecteurs de valorisation. 


\section{Conclusion}

On peut dire que les enseignants abordent leur activité sur la plate-forme en interrogeant la question de la motivation. L'engagement des étudiants n'est pas seulement un état d'esprit, il est le fruit d'une activité de réitération des consignes dans un réseau d'informations, une répétition qui permet aux apprenants d'intégrer les attendus de formation ${ }^{16}$. Si les enseignants qui travaillent sur Enfase considèrent que c'est en partie à l'étudiant de solliciter le partage, ils valorisent leur engagement pédagogique autour des activités d'explicitation et de diffusion des informations. Cet investissement éducatif concerne aussi bien les contenus disciplinaires que l'échange et la compréhension des consignes par les apprenants. Sur la plate-forme, les enseignants découvrent ainsi qu'ils peuvent œuvrer entre les connexions, faire se réunir les niveaux, organiser leurs enseignements en étoile. Ils ont alors la possibilité de montrer aux étudiants qu'ils peuvent à leur tour connecter entre elles les informations mises en ligne, et ne pas seulement s'en tenir au volume des connaissances transmises.Bien que ces informations soient très éclairantes, leur portée doit être relativisée vu la particularité du dispositif pédagogique encadrant le e-learning à Lille 3. Pour l'instant, il ne semble pas émerger, du moins en sciences de l'éducation, un modèle général d'enseignement $e$ learning. Mais cette absence de généralisation et de construction sur mesure n'a rien de déroutant dans le cadre de la formation pour adultes (Carré et Caspar, 2004) ou de la formation à distance (Giles, 1999), car le e-learning hérite encore régulièrement aujourd'hui de conceptions utilisées en présentiel.

\section{Références}

Albéro, B. (2004). Technologies et formation : travaux, interrogations, pistes de réflexion dans un champ de recherche éclaté. Savoirs Revue internationale de recherches en éducation et formation des adultes, $5,11-72$.

Ally, M. (2004). Foundations of educational theory for online learning. Dans T. Anderson et F. Elloumi (dir.), Theory and practice of online learning (p. 3-31). Athabasca, Canada : Athabasca University. Récupéré du site du Center for Distance Education de l'Université Athabasca : http://cde.athabascau.ca/online_book
Blandin, B. (2006). Comprendre et construire les environnements d'apprentissage. Note de synthèse pour l'habilitation à diriger des recherches, Université Paris X - Nanterre.

Carré, P. et Caspar, P. (2004). Traité des sciences et techniques de la formation. Paris : Dunod.

Cavanaugh, C. S. (2001). The effectiveness of interactive distance education technologies in K-12 learning: A meta-analysis. International Journal of Educational Telecommunications, 7(1), 73-88. Récupéré du site Education and Information Technology Library : http:/ / www.editlib.org

Cavanaugh, C., Gillan, K. J., Kromrey, J., Hess, M. et Blomeyer, R. (2004). The effects of distance education on K-12 student outcomes: A meta-Analysis. Naperville, IL : Learning Point Associates. Récupéré du site du North Central Regional Educational Laboratory, section Technology Home : http:// www.ncrel.org/ tech/distance

Giles, I. M. (1999). An Examination of Persistence and Dropout in the Online Computer-Conferenced Classroom. Doctoral Dissertation. Virginia Polytechnic Institute and State University, Northern Virginia Center.

Fenouillet, F. (2003). Motivation, mémoire et pédagogie. Paris : L'Harmattan.

Fenouillet, F. et Déro, M. (2006). Le e-learning est-il efficace? Savoirs - Revue internationale de recherches en éducation et formation des adultes, 12, 87-100.

Haeuw, F., Duveau-Patureau, V., Bocquet, F., Schaff, J.-L. et RoyPicardi, D. (2001, septembre). COMPETICE, outil de pilotage par les compétences des projets TICE dans l'enseignement supérieur. Ministère français de l'Éducation nationale, Direction de la Technologie, bureau B3. Récupéré du site Centre INFFO : http:/ / www.centreinffo.fr/IMG/pdf_competice9.pdf

Olson, T. M. et Wisher, R. A. (2002). The effectiveness of Web-based instruction: An initial inquiry. International Review of Research in Open and Distance Learning, 3(2). Récupéré de http: / / www.irrodl. org/index.php/irrodl/issue/view/14

Russell, T. L. (2001). The no significant difference phenomenon: A comparative research annotated bibliography on technology for distance education (5e éd.). Montgomery, AL : International Distance Education Certification Center.

Spencer, D. H. et Hiltz, S. R. (2001). Studies of ALN: An empirical assessment. Dans Proceedings of the 34th Hawaii International Conference on System Sciences (vol. 1, p. 1007). Washington, DC : IEEE Computer Society Press. 
U.S. Department of Education, National Center for Education Statistics, National Science Foundation, Division of Science Resources Statistics. (2002). The Application and Implications of Information Technologies in Postsecondary Distance Education: An Initial Bibliography, NSF 03-305, Project Director, Eileen L. Collins (Arlington, VA 2002).

Waxman, H. C., Lin, M.-F. et Michko, G. M. (2003). A meta-analysis of the effectivenesss of teaching and learning with technology on student outcomes. Naperville, IL : Learning Point Associates. Récupéré du site du North Central Regional Educational Laboratory, section Technology Home : http: / / www.ncrel.org/tech/effects2

\section{Notes}

1 Nous pensons notamment à la confidentialité des premiers processus de partage des savoirs universitaires par Internet, due en partie à la complexité et à la mise en application de technologies qui puissent épauler des projets pédagogiques conformes aux règles de la validation.

2 Nous pensons notamment à des revues comme Géographie de l'école ou Repères et Références statistiques de la DEP - Ministère de l'Éducation nationale.

3 En règle générale, à partir du 11e lien (10 liens par page sur Google, par exemple), nous constations une perte complète de pertinence.

4 Ici, la salle de cours est équipée soit d'un ordinateur, soit d'un outil de projection et de sonorisation, et les enseignants enrichissent alors leurs cours par la projection.

5 Par «présentiel quasi inexistant », nous entendons une formation qui soit ouverte et à distance, voire à longue distance. L'essentiel de la formation se fait en dehors de la présence de l'enseignant. Les étudiants ne se déplacent que pour l'évaluation finale. C'est par la plate-forme que le suivi s'effectue. L'organisation des enseignements tend alors à être pensée pour offrir à distance les potentialités habituelles d'un campus.

6 Foire aux questions.

7 Les données chiffrées ont été communiquées par les secrétariats et les responsables e-learning. Elles ont été ensuite croisées avec les statistiques inscrites sur le site Internet du ministère de l’Éducation nationale.

8 Besançon est la seule université de notre échantillon à n'avoir pas répondu à notre questionnaire. Nous ne pouvons donc commu- niquer à son sujet que les informations obtenues auprès de son secrétariat ou les données du ministère de l'Éducation nationale. Malgré de multiples tentatives, nous n'avons pu obtenir de l’Université Paris 8 aucune des informations présentées dans ce tableau.

10 On entend par « présentiel enrichi » l'usage de supports multimédias. Les salles de cours sont équipées d'un ordinateur, d'un outil de projection et de sonorisation. Cela concerne aussi bien le champ des ressources textuelles, graphiques, audio et vidéo, que des télé-expérimentations en direct (par exemple une expérience de chimie) ou encore des téléconférences visio et audio.

Dans le cas du «présentiel amélioré », les enseignants et les étudiants ont une boîte à lettres électronique. Avant et après le cours, l'enseignant met à la disposition des étudiants un certain nombre de ressources (exercices, fiches méthodologiques, accès aux supports pédagogiques utilisés pendant le cours, accès à des dispositifs d'autoévaluation). Il s'agit d'une stratégie documentaire et de communication.

Le "présentiel allégé » concerne le partage d'apprentissages s'effectuant en dehors de la présence physique du formateur. Quelques heures de cours ou de TD sont remplacées par des activités d'autoformation multimédia (ou non) qui sont planifiées par l'enseignant, mais peuvent être tutorées par d'autres personnes (tuteurs, accompagnateurs d'entreprises, anciens...).

On parlera de «présentiel réduit » lorsque l'essentiel de la formation se fait en dehors de la présence de l'enseignant. Celui-ci contractualise les objectifs en début de formation avec l'étudiant; il intervient de façon synchrone ou asynchrone, en présence ou à distance, essentiellement pour préciser ou réexpliquer différemment des notions, animer des temps de partage et d'échange, mais aussi pour évaluer. Il s'agit d'une stratégie de création d'un environnement pédagogique soit de type physique (centre de ressources), soit virtuel (environnement d'apprentissage).

Le «présentiel quasi inexistant » est la formation ouverte et à distance ou à longue distance. L'essentiel de la formation se fait en dehors de la présence de l'enseignant. Les étudiants ne se déplacent que pour l'évaluation finale. Une plate-forme permet le suivi des étudiants et offre à distance les potentialités habituelles d'un campus (voir Haeuw et al., 2001).

11 La licence «mixte » des Sciences de l'éducation de Paris X, 
réservée notamment aux personnes exerçant une activité professionnelle ou en congé parental d'éducation, se déroule sur deux ans. Les cours sont dispensés le samedi à l'Université, complétés par un enseignement à distance.

12 Les réflexions sont le fruit d'une synthèse de propos recueillis lors d'entretiens avec différents enseignants du dispositif $e$-learning Enfase de Lille 3.

13 Sessions de questions/réponses avec les étudiants en fin de cours, échanges épisodiques dans les couloirs ou au secrétariat, comptes rendus rapides pendant les permanences lors des remises de notes, etc.

14 Ce sont des logiciels qui permettent de créer des exercices en ligne. Ils sont principalement utilisés dans le cadre des Travaux dirigés, pour observer et vérifier la progression des acquis chez les étudiants.

15 Soit en chronométrant électroniquement le temps de renseignement pour chaque étudiant, soit en rendant actif le QCM pour une durée limitée seulement.

16 « D’une manière générale, tout ce qui encourage la compétence perçue va augmenter la motivation intrinsèque et, à l'inverse, tout ce qui altère la perception de compétence va diminuer la motivation intrinsèque. Cependant, cela ne veut pas forcément dire que les feedbacks négatifs vont toujours altérer notre perception de compétence, ni un feedback positif l'augmenter. . . Le contexte, contrôlant ou informant, où survient une information, détermine pour une grande part la manière dont cette information modifie la motivation intrinsèque. Un feedback négatif dans une situation informante va donc être perçu comme l'occasion de corriger une erreur de fonctionnement comportemental. » (Fenouillet, 2003, p. 65) 